\title{
UNIQUE FACTORIZATION IN A PRINCIPAL RIGHT IDEAL DOMAIN ${ }^{1}$
}

\author{
R. E. JOHNSON
}

An integral domain with unity in which every right ideal is principal is called a pri-domain. The classical example of a pri-domain is the polynomial domain $F[x]=\left\{\sum x^{i} a_{i} \mid a_{i} \in F\right\}$ over a division ring $F$, where multiplication is defined by $a x=x a^{\sigma}+a^{\delta}, a \in F$, with $\sigma$ a monomorphism and $\delta$ an associated derivation of $F$ (see Ore, [1]).

In what follows, $R$ is a pri-domain and $L$ is its lattice of right ideals. Each $a \in R$ has dimension, $\operatorname{dim} a$, defined to be the length of the longest chain in the interval $[a R, R]$ of $L$. The elements of dimension 1 are the primes of $R$. Although $\operatorname{dim} a$ is conceivably infinite ${ }^{2}$ for some nonzero $a \in R$, we are only interested in those elements for which $\operatorname{dim} a<\infty$. For convenience, let $R^{*}=\{a \in R \mid a \neq 0,0<\operatorname{dim} a$ $<\infty\}$.

Each $a \in R^{*}$ may be expressed as a product of primes, $a=p_{1} \cdot p_{2}$ - ... $p_{n}$, where $n=\operatorname{dim} a$. For if $p_{1}$ is a prime left factor of $a$ and $a=p_{1} a_{1}$, then $R-a_{1} R \cong p_{1} R-a R$ (as right $R$-modules) and therefore $\operatorname{dim} a_{1}=n-1$. The factorization now follows by induction. Elements $b$ and $c$ of $R^{*}$ are said to be similar, $b \sim c$, if $R-b R \cong R-c R$. It may be shown by the usual proof for pri- and pli-domains (see $[2$, p. 34]) that if $a=p_{1} \cdot p_{2} \cdot \cdots \cdot p_{n}=q_{1} \cdot q_{2} \cdot \cdots \cdot q_{n}$, where all $p_{i}$ and $q_{i}$ are primes, then there exists a permutation $\alpha$ of $(1,2, \cdots, n)$ such that $q_{i} \sim p_{\alpha(i)}, i=1,2, \cdots, n$.

Whenever $a \in R$ is represented as a product $a=a_{1} \cdot a_{2} \cdot \cdots \cdot a_{n}$, then $a$ can also be represented as a product

$$
a=\left(a_{1} u_{1}\right)\left(u_{1}^{-1} a_{2} u_{2}\right) \cdots\left(u_{n-1}^{-1} a_{n}\right)
$$

for any units $u_{1}, u_{2}, \cdots, u_{n-1}$ of $R$. Let us call a factorization $a=a_{1} \cdot a_{2} \cdot \cdots \cdot a_{n}$ of $a$ as a product of elements of a stated type unique if every other representation of $a$ as a product of elements of the stated type has the form (1) above. It is the purpose of this note to describe a particular type of unique factorization that occurs in $R$.

Associated with each $a \in R^{*}$ is the subset $L_{a}$ of $L$ defined by

$$
L_{a}=\{B \in[a R, R] \mid[a R, R]=[a R, B] \cup[B, R]\} .
$$

By a lattice-theoretic argument, it is easily demonstrated that $L_{a}$ is

Received by the editors, December 18, 1963.

${ }^{1}$ Research supported by NSF grant G24155.

2 No example is known to the author where $\operatorname{dim} a=\infty$. 
a finite chain $a R=B_{k}<B_{k-1}<\cdots<B_{0}=R$ and that

$$
[a R, R]=\left[B_{k}, B_{k-1}\right] \cup\left[B_{k-1}, B_{k-2}\right] \cup \ldots \cup\left[B_{1}, B_{0}\right] .
$$

Let us call $a \in R^{*}$ simple if $L_{a}=\{a R, R\}$. Clearly every prime is simple, and if $a$ is simple and $b \sim a$, then $b$ is also simple. If $R$ is commutative, then $a \in R^{*}$ is simple if and only if either $a$ is prime or $a$ has two dissimilar prime factors.

If $a \in R^{*}$ and $L_{a}=\left\{B_{0}, B_{1}, \cdots, B_{k}\right\}$ as above, with $B_{i}=b_{i} R$ and $b_{i}=b_{i-1} a_{i}, i=1,2, \cdots, k, b_{0}=1$ and $b_{k}=a$, then $B_{i-1}-B_{i} \cong R-a_{i} R$, $i=1,2, \cdots, k$. Since the lattice $\left[B_{i}, B_{i-1}\right]$ is not a union of two proper intervals of $L$, evidently each $a_{i}$ is simple. Thus, $a=a_{1} \cdot a_{2} \cdot \ldots$ - $a_{k}$ where each $a_{i}$ is simple but no element of the form $a_{i} \cdot a_{i+1} \cdot \ldots$ $\cdot a_{j}, i<j$, is simple. It is easy to see that this is the unique factorization of $a$ associated with the lattice $L_{a}$.

Let $a \in R^{*}$ and $a=a_{1} \cdot a_{2} \cdot \cdots a_{k}$ be the unique factorization of $a$ associated with $L_{a}$ as above. If $a_{1}=g \cdot h$ for some $g, h \in R^{*}$ and $c=h \cdot a_{2} \cdot \cdots \cdot a_{k}$, then $g c=a$. Since $[c R, R] \cong[a R, g R]$ and $[a R, g R]$ $>\left[a R, a_{1} R\right]$, evidently $[c R, R]=\left[c R, c_{k-1} R\right] \cup\left[c_{k-1} R, c_{k-2} R\right] \cup \ldots$ $\cup\left[c_{1} R, R\right]$ where $c_{i}=h \cdot a_{2} \cdot \cdots \cdot a_{i}, i>1$, and $c_{1}=h$. Each of the intervals $\left[c_{i} R, c_{i-1} R\right]$ is irreducible (i.e., not the union of two intervals) with the possible exception of $\left[c_{1} R, R\right]$. Hence, the factorization of $c$ associated with $L_{c}$ has the form $c=h_{1} \cdot \cdots \cdot h_{r} \cdot a_{2} \cdot \cdots \cdot a_{k}$ where $h=h_{1} \cdot \cdots \cdot h_{r}$ is the factorization of $h$ associated with $L_{h}$.

A factorization of $a \in R^{*}$ into simple elements $a=a_{1} \cdot a_{2} \cdot \cdots \cdot a_{k}$ is called irredundant if no sub-product $a_{i} \cdot a_{i+1} \cdot \cdots \cdot a_{j}, i<j$, of $a$ is simple. The main result of the paper is as follows.

TheOREM. Each $a \in R^{*}$ has a unique irredundant factorization into simple elements.

Proof. The theorem is true if $\operatorname{dim} a=1$. Assume that the theorem is true for every element of $R^{*}$ of dimension less than $n$, and let $a \in R^{*}$ have dimension $n>1$. We know that $a$ has an irredundant factorization into simple elements associated with $L_{a}$, say $a=a_{1} \cdot a_{2} \cdots \cdots a_{k}$. If $k=1$, then the factorization clearly is unique, so let us assume that $k>1$. Also assume that $a=c_{1} \cdot c_{2} \cdot \cdots \cdot c_{m}, m>1$, is an irredundant factorization of $a$ into simple elements.

Since $c_{1} R \in[a R, R]=\left[a R, a_{1} R\right] \cup\left[a_{1} R, R\right]$, either $c_{1} R<a_{1} R$ or $c_{1} R \geqq a_{1} R$. If $c_{1} R<a_{1} R$ then $\left[c_{1} R, R\right]=\left[c_{1} R, a_{1} R\right] \cup\left[a_{1} R, R\right]$ contrary to the simplicity of $c_{1}$. Hence, $c_{1} R \geqq a_{1} R$. If $c_{1} R=a_{1} R$ then $a$ has unique factorization by induction.

Finally, if $c_{1} R>a_{1} R$ then $a_{1}=c_{1} h$ for some $h \in R^{*}$ and $b=c_{2} \cdots \cdots c_{m}$ $=h \cdot a_{2} \cdot \cdots \cdot a_{k}$. By a previous remark, the irredundant factorization 
of $b$ into simple elements associated with $L_{b}$ has the form $b=h_{1}$. . . $\cdot h_{r} \cdot a_{2} \cdot \cdots a_{k}$. Since $\operatorname{dim} b<n$, this must be the unique factorization of $b$ into simple elements. Therefore, $c_{2} \cdot \cdots \cdot c_{r+1}=h_{1} \cdot \cdots \cdot h_{r} \cdot u$ for some unit $u$. However, then $c_{1} \cdot c_{2} \cdots \cdots c_{r+1}=a_{1} \cdot u$ contrary to the assumption that $c_{1} \cdot c_{2} \cdot \cdots \cdot c_{r+1}$ is not simple. This proves the theorem.

Let us call $a \in R^{*}$ primary if $[a R, R]$ is a chain. The above theorem has the following form for primary elements.

Corollary. An element a of $R^{*}$ is primary if and only if a has a unique representation as a product of primes.

If $R$ is commutative, then our definition of primary agrees with the usual one; $a \in R^{*}$ is primary if $a=p^{n} u$ for some prime $p$ and some unit $u$.

Our results on simple elements of $R^{*}$ are too fragmentary to present at this time.

We close this note with an example. Let $F=Z_{2}(t)$ be a transcendental extension of the integers modulo $2, R=F[x]=\left\{\sum x^{i} a_{i} \mid a_{i} \in F\right\}$, $F \rightarrow^{\sigma} F$ be the monomorphism $a^{\sigma}=a^{2}$, and $\delta=0$. Thus, $a x=x a^{2}$ for every $a \in F$. The ring $R$ is a pri-domain, but not a pli-domain. An unusual feature of $R$ is that $x+a \sim x+b$ for all nonzero $a, b \in F$. The following statements can be verified by some elementary computa-

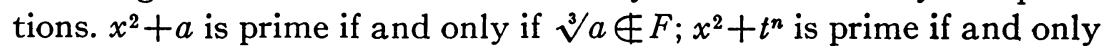
if $(n, 3)=1$; if $a=b^{3} \neq 0$, then $x^{2}+a$ has the unique factorization $x^{2}+a=(x+b)(x+a / b)$; thus, $x^{2}+a$ is always primary; $(x+a)^{2}$ is not primary if $a+a b=b^{2} \neq 0$ for some $b \in F$, for then $(x+a)^{2}$ $=(x+b)\left(x+a^{2} / b\right) ; x^{2}+x t$ is primary; $(x+1)\left(x^{2}+t\right)$ is primary.

\section{BIBLIOGRAPHY}

1. O. Ore, Theory of non-commutative polynomials, Ann. Math. 34 (1933), 480508.

2. N. Jacobson, The theory of rings, Math Surveys No. 2, Amer. Math. Soc., Providence, R. I., 1943.

UNIVERSITY OF ROCHESTER 